\title{
ABOUT THE APPLICATION OF ANGLE-ACTION \\ VARIABLES TO THE ROTATION OF DEFORMABLE CELESTIAL BODIES
}

\author{
YURI V. BARKIN AND JOSE M. FERRANDIZ \\ Depto. Matemática Aplicada \\ Universidad de Alicante, 03080 Alicante. Spain \\ AND \\ JUAN GETINO \\ Grupo de Mecánica Celeste \\ Facultad de Ciencias, 47005 Valladolid. Spain \\ e-mail: getino@hp9000.uva.es
}

\begin{abstract}
We begin with the development of a new analytical theory for the rotational motion of deformable celestial bodies. In this work we present a new unperturbed Hamiltonian which includes the main term affecting the Chandler period.
\end{abstract}

\section{Motivation of this paper}

The effect of the elasticity of the mantle on the Earth's rotation has been developed by Getino and Ferrándiz $(1991,1995)$ in a Hamiltonian framework, using a set of Andoyer-like canonical variables, $\lambda, \mu, \nu, \Lambda, M, N$ (and the auxiliary parameters $\sigma, I)$. As described in Getino and Ferrándiz (1995), the main contribution to the Chandler period arises from the term $T_{r}$, that is to say, the increment of the kinetic energy due to the centrifugal deformation (caused by the own rotation of the Earth). So that, the principal ideas of this work are, on the one hand, to consider a new expression of the Hamiltonian in such a way that the new unperturbed Hamiltonian includes the main effects affecting the Chandler period, in order to make its study easier, and on the other hand, to develop an analytical theory suitable to 
be applied to more general celestial bodies, when the principal moments of inertia are in the form $A \neq B \neq C$.

Let us break down the term $T_{r}$ into two parts, $T_{r}=T_{r}^{0)}+T_{r}^{1)}$. From Getino and Ferrándiz (1991), the main part $T_{r}^{0)}$ has the expression:

$$
T_{r}^{0)}=\frac{3}{2} D_{r} \frac{M^{2}-N^{2}}{C}\left[\frac{\sin ^{2} v}{A}+\frac{\cos ^{2} v}{B}\right],
$$

where $D_{r}$ is a coefficient depending on the internal structure of the body (for the case of the Earth, $D_{r}=-2.845379 \times 10^{41}$ c.g.s.), and $A, B$ and $C$ are the principal moments of inertia including the effect of the centrifugal deformation in the form $A \simeq A_{0}+D_{r}, B \simeq B_{0}+D_{r}, C \simeq C_{0}-2 D_{r}$, where $A_{0}, B_{0}, C_{0}$ correspond to the body in the absence of deformation (Getino and Ferrándiz, 1991).

Now, if we define new principal moment of inertia $\tilde{A}, \tilde{B}, \tilde{C}$ in the form

$$
\frac{1}{\tilde{A}}=\frac{1}{A}\left(1+\frac{3 D_{r}}{C}\right), \frac{1}{\tilde{B}}=\frac{1}{B}\left(1+\frac{3 D_{r}}{C}\right), \frac{1}{\tilde{C}}=\frac{1}{C},
$$

the new unperturbed Hamiltonian has the following expression

$$
H_{0}=T_{0}+T_{r}^{0)}=\frac{M^{2}-N^{2}}{2}\left(\frac{\sin ^{2} \nu}{\tilde{A}}+\frac{\cos ^{2} \nu}{\tilde{B}}\right)+\frac{N^{2}}{2 \tilde{C}},
$$

and we can conclude that:

"The free rotational motion of a celestial body deformed by its own rotation is described in first approximation by the solution of the EulerPoinsot problem corresponding to a rigid body with special principal moments $\tilde{A}, \tilde{B}, \tilde{C} "$.

Thus, this solution will explain the Chandler period. The analytical integration of (1) by means of action-angle variables, as well as the development of the of the perturbations due to the force function of the Earth-Moon system is in progress, and we hope to present the obtained results soon.

\section{Acknowledgments}

This work has been partially supported by CICYT, Project No. ESP93741.

\section{References}

Getino, J. and Ferrándiz, J.M.: 1991, Celes. Mech., 51, 35-65.

Getino, J. and Ferrándiz, J.M.: 1995, Celes. Mech., 61, 117-180. 